Kompass

\section{Autoimmun}

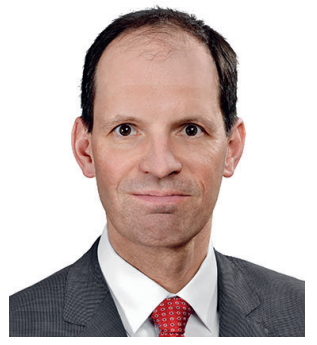

Martin Ahringer

Medizinische Klinik und Poliklinik III, Universitätsklinikum Carl Gustav Carus der TU Dresden, Dresden, Deutschland

Sehr geehrte Damen und Herren, liebe Kolleginnen und Kollegen,

in Anbetracht der COVID-19-Situation ist es gar nicht einfach, auf systemische Autoimmunerkrankungen zu fokussieren - aber gleichzeitig haben gerade diese Patientinnen und Patienten momentan große Sorgen und benötigen eine besondere Betreuung. Auch wenn die bisherigen Erkenntnisse nicht für eine besonders ausgeprägte Risikoerhöhung durch die Immunmodulation und -suppression sprechen, fehlen uns belastbare Daten. Diese Unwissenheit macht unsicher. Je besser wir die Erkrankung verstehen, desto einfacher wird es sein, Patientinnen und Patienten optimal zu beraten.

Das gleiche gilt auch für die systemischen Autoimmunerkrankungen, vielleicht in den letzten Jahrzehnten besonders für den systemischen Lupus erythematodes (SLE). Umso erfreulicher sind die Entwicklungen des letzten Jahres. So klar waren die EULAREmpfehlungen noch nie. Erstmals ist ausformuliert, dass alle SLE-Patientinnen und -Patienten Hydroxychloroquin bekommen sollten. Die EULAR-Empfehlungen setzen zudem das Treat-to-target-Prinzip für den SLE um, das auf den Seiten 45-51 diskutiert wird. Das Ziel ist die Remission. Tritt im weiteren Verlauf eine Organentzündung auf, muss diese kontrolliert werden - aber ohne Dauerglukokortikoide von mehr als 5 mg Prednisolonäquivalent täglich. Damit gibt es für den Großteil der Patienten einfache Handlungsempfehlungen.

Aus meiner Sicht gilt das auch für die EULAR/ACR 2019-Klassifikationskriterien für den SLE. Die antinukleären Antikörper (ANA) nehmen nun die Position eines Eingangskriteriums ein. Patientinnen und Patienten, die

\title{
Systemischer Lupus erythematodes 2020 - besseres Verständnis vereinfacht Management
}

nie ANA hatten, können nicht klassifiziert werden, aber das kommt nur sehr selten vor. Die Liste an Einzelkriterien ist übersichtlich und entspricht inhaltlich den bisherigen Kriterien, nur nicht infektiöses Fieber ist als wichtiges Frühsymptom dazugekommen und die Lymphopenie kommt wegen ihrer geringen Spezifität nicht mehr vor. Die Kriterien sind in 10 Organdomänen geordnet und gewichtet, mit einem Cut-off von 10, den nur die proliferative Lupusnephritis Klasse III oder IV alleine erreicht. Und es gibt eine Regel für alle Kriterien: Gezählt wird nur, was nicht vermutlich eine andere Ursache als den SLE hat. Auch die neuen SLE-Kriterien sind relativ einfach, weil wir die Erkrankung mittlerweile besser verstehen.

Schließlich vereinfachen drei positive klinische Prüfungen unsere immunologische Sicht des SLE. Anifrolumab weist mit einer positiven Phase-III-Studie endlich schlüssig nach, dass Typ I-Interferone wie Interferon-a eine Rolle beim SLE spielen. Anifrolumab ist ein monoklonaler Antikörper, der den gemeinsamen Rezeptor für alle Typ I-Interferone blockiert. Dass diese als Antwort auf Immunkomplexe durch plasmazytoide dendritische Zellen freigesetzt werden, ist schon lange bekannt, aber nun folgt auf die Theorie der Therapieansatz. Immunkomplexe bringen auch Monozyten dazu, das B-ZellZytokin BLyS/BAFF zu produzieren, das wieder autoimmune B-Zellen fördert. Der AntiBLyS/BAFF-Antikörper Belimumab ist für die Behandlung des nicht renalen SLE eine Routine-Option. Jetzt hat eine Lupus-NephritisStudie gezeigt, dass die BLyS/BAFF-Blockade auch bei der Lupusnephritis hilft. Und in einer weiteren Lupus-Nephritis-Studie hat Obinutuzumab, ein neuer B-Zellen depletierender Anti-CD20-Antikörper, Erfolg gehabt.
Damit sind B-Zellen, Belimumab und die Typ-I-Interferone als Faktoren gesichert und zumindest für BLyS/BAFF Lupusnephritis und nicht renaler SLE zusammengeführt. Auch wenn der SLE im Titel steht, und der nephrologische Wissenstransfer zu Chancen durch Biopsie-geführtes Management der Lupusnephritis sowie der Fallbericht von Dan Gheorghe AC et al. (Seite 77-80) diesen Schwerpunkt vertiefen, beinhaltet dieses Heft noch andere spannende Themen: Die Rolle der endokrinen Transition spielt für viele Autoimmunerkrankungen eine wesentliche Rolle - beim SLE ist das Verhältnis Patientinnen zu Patienten ja etwa 10:1. Die B-Zell-Depletion führt vom SLE weiter zur Multiplen Sklerose. Dahingegen sind für die autoimmunen Nebenwirkungen der Checkpoint-Inhibitoren, die uns alle zunehmend beschäftigen, T-Zellen wesentlich. In diesem Heft finden Sie Artikel zur Lungentoxizität und zum Effekt der Photopherese auf die Colitis ulcerosa bei Therapie mit diesen immunologischen Krebsmedikamenten.

Falls Sie in den nächsten Wochen wegen COVID-19 keine Zeit haben sollten, die Artikel zu lesen, hoffe ich, das Heft ist spannend genug, um bis danach aufgehoben zu werden. Hoffentlich wird das nicht allzu lange dauern. Ich wünsche Ihnen allen, dass Sie gut durch die COVID-Epidemie kommen. Alles Gute!

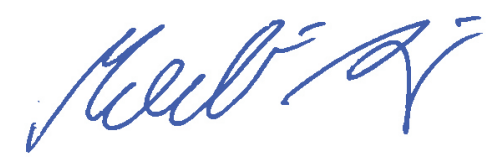

Prof. Dr. Martin Aringer information@karger.com

www.karger.com/kai

(๑) 2020 S.Karger GmbH, Freiburg

Karger ${ }^{\prime \prime}=$
Prof. Dr. Martin Aringer

Medizinische Klinik und Poliklinik III

Universitätsklinikum Carl Gustav Carus der TU Dresden

Fetscherstraße 74, 01307 Dresden, Deutschland

martin.aringer@uniklinikum-dresden.de 